\title{
MOSAICS OF COMPACT METRIC SPACES $\left({ }^{1}\right)$
}

\author{
BY \\ WALTER F. DAVISON
}

Introduction. Our purpose is to study a class of topological spaces which is more general than the class of first countable Hausdorff spaces and less general than the class of $k$-spaces. To construct a space $(X, J)$ of this class, called a mosaic space, there is employed a collection of topological spaces $\left\{\left(X_{a}, J_{a}\right): a \in A\right\}$ which cover $X$ and which serve as "test sets" to determine the topology 3 . Since each space $\left(X_{a}, J_{a}\right)$ is to be thought of as the prototype of a fairly "geometrical" object such as an arc, curve, or simplex, we require that each $\left(X_{a}, J_{a}\right)$ be a compact metric space; in order to guarantee that each such compact metric space be a subspace of $(X, \Im)$ the former are "patched together" in a suitable fashion. After establishing the definition of a mosaic space and some basic properties, we show that among the topological spaces it is precisely the mosaic spaces which are in one-to-one correspondence with the Kuratowski $\mathscr{L}^{*}$-spaces which have the additional feature that limits are unique. By generalizing the mosaic concept by "patching together" functions we find that a closed continuous image of a mosaic space is a mosaic space; that a quasi-compact image of a mosaic space is again a mosaic space, provided limits of convergent sequences are unique in the image space; and that every mosaic space is the quasi-compact image of a suitable locally compact metric space.

The latter half of the paper gives necessary and sufficient conditions for a mosaic space to be hereditary, that is, for each subspace to be a mosaic space; for a mosaic space to be countably compact; and for the Cartesian product of a fixed mosaic space with an arbitrary mosaic space to be again a mosaic space. Further it is shown that a mosaic space can always be countably compactified so as to remain a mosaic space, and necessary and sufficient conditions are given for a mosaic space to be embedded in a compact mosaic space. Also we give an example of a compact hereditary mosaic space which is not a Hausdorff space. Finally, some results are obtained for function space topologies within the mosaic context.

1. Definition and properties of mosaic spaces. There will frequently arise topologies $\mathfrak{I}$ which are defined on subsets $Y$ of a set $X$ rather than on $X$ itself. Moreover, closed sets will be of greater importance than open sets. We thereby adopt for the expression " $E \in J$ " the following definition: for every

Presented to the Society, December 27, 1955 under the title Convergent sequences and mosaics; received by the editors March 18, 1957 and, in revised form, November 6, 1957.

(1) A portion of this material is to be found in a thesis submitted to the University of Virginia. 
$E \subset X, E \in J$ if and only if $E$ is a J-closed subset of the topological space $(Y, \Im)$. The expression " $R<S$ " will denote the fact that $R$ is a subsequence of the sequence $S$, " $S_{j}$ " will denote the $j$ th term of the sequence $S$, and " $S_{J}$ " will denote the point set of the sequence $S$. For a given topology $\mathcal{J}$, to say that a sequence $S$ converges $(\Phi(J))$ to a point $x$ means that $S$ converges to $x$ under the usual definition of convergence according to the topology $\Im$. By definition a topology $J$ on $X$ is full if and only if the following statement holds: for all $E \subset X$, if $E \notin J$ then there exists a sequence $S$ and a point $x$ in $X$ such that $S_{J} \subset E, x \in E$ and $S$ converges $(\Phi(J))$ to $x$. Any terminology introduced without explanation will be that employed by Kelley [7].

1.1. Definition. A collection $\left\{\left(X_{a}, J_{a}\right): a \in A\right\}$ is a mosaic of topological spaces on a set $X$ if and only if (i) each $\left(X_{a}, J_{a}\right)$ is a topological space; (ii) $X$ $=\mathrm{U}\left\{X_{a}: a \in A\right\}$; and (iii) the following compatibility condition is satisfied: for all $E \subset X$ and $a, b \in A$, if $E \in J_{a}$ then $E \cap X_{b} \in J_{b}$. For a mosaic of topological spaces on $X$ the mosaic topology $J$ is defined as follows: for all $E \subset X$, $E \in J$ if and only if $E \cap X_{a} \in J_{a}$ for all $a \in A$. If each $\left(X_{a}, J_{a}\right)$ is a compact metric space then the topological space $(X, J)$ determined by the collection $\left\{\left(X_{a}, J_{a}\right): a \in A\right\}$ will be called simply a mosaic space, for the sake of brevity.

Implicit in the definition is the first lemma.

1.2. Lemma. Let $\left\{\left(X_{a}, J_{a}\right): a \in A\right\}$ be a mosaic of topological spaces on $X$ and let $\Im$ be the mosaic topology. Then for all $a \in A,\left(X_{a}, \Im_{a}\right)$ is a closed subspace of $(X, J)$.

From (1.1) and (1.2) it is immediate that cvery mosaic space is a $k$-space.

1.3. Theorem. The topology $\mathfrak{I}$ of a mosaic space is full.

Proof. Let $\left.\left\{X_{a}, \Im_{a}\right): a \in A\right\}$ be the mosaic of compact metric spaces which determines the topology $\Im$. Let $E \subset X$ and assume that $E \notin J$ J Then by definition there exists an $a \in A$ such that $E \cap X_{a} \notin J_{a}$. Since $\left(X_{a}, J_{a}\right)$ is metric there exists a sequence $S$ and a point $x$ in $X$ such that $S_{J} \subset E \cap X_{a}, x \notin E \cap X_{a}$, and $S$ converges $\left(\Phi\left(\mathcal{J}_{a}\right)\right)$ to $x$. Thus $S_{J} \subset E, x \notin E$, and $S$ converges $(\Phi(\mathfrak{J}))$ to $x$ by (1.2).

1.4. Lemma. Let $(X, J)$ be a mosaic space determined by the mosaic of compact metric spaces $\left\{\left(X_{a}, \Im_{a}\right): a \in A\right\}$. Then a subset $E \subset X$ has a J-accumulation point if and only if there exists an $a \in A$ such that $E \cap X_{a}$ is infinite.

Proof. If $E \cap X_{a}$ is infinite for some $a \in A$ then it has a $\mathfrak{I}_{a}$-accumulation point, since $\left(X_{a}, J_{a}\right)$ is compact metric. But $\left(X_{a}, J_{a}\right)$ is a subspace of $(X, \Im)$ by (1.2), therefore $E$ has a J-accumulation point. Conversely, if $E \cap X_{a}$ is finite for all $a \in A$ then $E$ and each subset of $E$ is 3 -closed. Therefore $E$ is discrete and has no 3 -accumulation point.

1.5. Lemma. In a mosaic space, if $S$ converges $(\Phi(J))$ to $x$ then there exists a sequence $S^{\prime}$ and an $a \in A$ such that $S^{\prime} \prec S$ and $S_{J}^{\prime} \cup\{x\} \subset X_{a}$. 
Proof. If $S_{J}$ is a finite set then the lemma is obvious. Alternatively, if $S_{J}$ is infinite then $x$ is a 3 -accumulation point of $S_{J}$, and by (1.4) there exists an $a \in A$ such that $S_{J} \cap X_{a}$ is infinite. From $S_{J} \cap X_{a}$ a sequence $S^{\prime}$ may be chosen such that $S^{\prime}<S$ and $S_{J}^{\prime} \subset X_{a}$. Since $X_{a} \in J$ by (1.2) we also have $x \in X_{a}$.

1.6. Theorem. In a mosaic space, limits of $\Phi(\mathfrak{J})$-convergent sequences are unique.

Proof. If $S$ converges $(\Phi(J))$ to $x$ and to $y$ then by (1.5) there exists an $a \in A$ and an $S^{\prime}<S$ such that $S_{J}^{\prime} \cup\{x\} \subset X_{a}$. Since $X_{a} \in J$ we also have $y \in X_{a}$. By (1.2) the sequence $S^{\prime}$ converges $\left(\Phi\left(J_{a}\right)\right)$ to $x$ and to $y$. Therefore $x=y$, since $\left(X_{a}, J_{a}\right)$ is metric.

Since obviously $\{x\} \cap X_{a} \in J_{a}$ for all $a \in A$ and every $x \in X$, a mosaic space is a $T_{1}$-space and countable compactness may be characterized as follows: for all $E \subset X, E$ is J-countably compact if and only if every infinite subset of $E$ has a 3 -accumulation point in $E$. For a set $X$ with a given class of convergent sequences $\mathscr{L}$ a subset $E \subset X$ is defined to be $\mathscr{L}$-sequentially compact if and only if every sequence in $E$ has a subsequence which $\&$-converges to a point in $E$.

1.7. Theorem. In a mosaic space, a subset $E$ is J-countably compact if and only if it is $\Phi(\mathfrak{J})$-sequentially compact.

Proof. That $\Phi(\mathfrak{J})$-sequential compactness implies J-countable compactness is known for general topological spaces, so we prove only the converse. Assume that $E \subset X$ is not $\Phi(J)$-sequentially compact, so that there exists a sequence $S$ such that $S_{J} \subset E$ and no subsequence of $S$ converges $(\Phi(J))$ to a point in $E$. If $S_{J}$ has no J-accumulation point then it trivially qualifies as the required infinite subset of $E$ which has no J-accumulation point in $E$. Alternatively, if $S_{J}$ has a J-accumulation point then by (1.4) there exists an $a \in A$ such that $S_{J} \cap X_{a}$ is infinite. Since $\left(X_{a}, J_{a}\right)$ is compact metric we may choose a sequence $S^{\prime}$ such that $S^{\prime} \prec S$ and $S^{\prime}$ converges $\left(\Phi\left(J_{a}\right)\right)$ to some $x \in X_{a}$. By (1.2) the sequence $S^{\prime}$ converges $(\Phi(J))$ to $x$, and so $x \notin E$ since $S^{\prime} \prec S$. At the same time $x$ is the only $J_{a}$-accumulation point of $S_{J}^{\prime}$ and $X_{a} \in J$ by (1.2), implying that $x$ is the only J-accumulation point of $S_{J}^{\prime}$. The set $S_{J}^{\prime}$ thereby qualifies as an infinite subset of $E$ with no J-accumulation point in $E$.

1.8. Theorem. In a mosaic space, every J-countably compact subset is Jclosed.

Proof. Assume that $E \notin J$, so that there exists an $a \in A$ such that $E \cap X_{a}$ $\notin J_{a}$. Because $\left(X_{a}, J_{a}\right)$ is a closed, compact metric subspace of $(X, J)$ there exists an infinite set in $E \cap X_{a}$ with no $J_{a}$-accumulation point in $E$, and thereby no $J$-accumulation point in $E$. Therefore $E$ is not countably compact.

1.9. Corollary. In a mosaic space, every J-compact subset is J-closed. 
Given a mosaic $\mathfrak{M T}_{0}$ of compact metric spaces on a set $X$ we have seen how to obtain the corresponding mosaic topology $\mathfrak{I}$ on $X$. Each compact metric space $\left(X_{0}, J_{0}\right)$ of the mosaic $\mathfrak{M}_{0}$ is embedded in the mosaic space $(X, \mathfrak{J})$ in which $J_{0}$ is simply $J$ relativized to $X_{0}$. However, there is no guarantee that every compact metric subspace of $(X, J)$ is a member of the originally given mosaic $\mathfrak{M}_{0}$, and in fact this need not be so. Nevertheless, by means of (1.9) we are assured that the collection of all compact metric subspaces of $(X, \Im)$ satisfies the compatibility condition, and so forms a mosaic $\mathfrak{T}$ of compact metric spaces which in general includes the mosaic $\mathfrak{M}_{0}$. If this enlarged mosaic $\mathfrak{T}$ is employed to obtain a new mosaic topology on $X$ then it is easily seen that this new topology is just $J$ itself. Beginning with a mosaic $\mathfrak{M}_{0}$ of compact metric spaces and iterating this procedure nothing new is obtained beyond the mosaic $\mathfrak{T}$, which we shall call a full mosaic of compact metric spaces. Each full mosaic of compact metric spaces corresponds to some one mosaic topology, and conversely. The result (1.9) must be regarded as important, as it eliminates the pathological possibility of obtaining by iteration a sequence of coarser and coarser mosaic topologies, alternating with a sequence of larger and larger mosaics.

2. Mosaic spaces and L-spaces. We now turn to the construction of mosaic spaces from sequence convergence classes, and to the resulting correspondence between mosaic spaces and certain convergence classes. For a fixed set $X$ let $\&$ be the collection of all convergence classes $\mathscr{L}$ with the following properties: (i) every constant sequence in $X$ converges $(\mathscr{L})$ to the point of which its point set is composed; (ii) for every sequence $S$ and every point $x$ in $X$, if $S$ converges (\&) to $x$ then every subsequence of $S$ converges (\&) to $x$. Thus $(X, \mathfrak{L})$ for $\mathscr{L} \in \mathbb{R}$ is a Frèchet $\mathscr{L}$-space [9, Chapter II]. Given a convergence class $\mathscr{L} \in \mathbb{R}$, a topology $\Psi(\mathfrak{L})$ on $X$ may be generated in the following standard manner: for all $E \subset X, E \in \Psi(\mathscr{L})$ if and only if every limit of every $\mathcal{L}$-convergent sequence in $E$ is also in $E$. From each $\mathcal{L} \in \mathbb{R}$ we obtain its *-extension $\mathfrak{L}^{*}$ by demanding for every sequence $S$ and point $x$ in $X$ that $S$ converges $\left(\mathscr{L}^{*}\right)$ to $x$ if and only if every subsequence of $S$ has a subsequence which converges $(\mathscr{L})$ to $x$. Let $\mathfrak{R}^{*}$ be the collection of all $\mathfrak{L}$-classes each of which is its own *-extension; thus $(X, \mathfrak{L})$ for $\mathscr{L} \in \mathfrak{R}^{*}$ is a Kuratowski $\mathfrak{L}^{*}$-space. Finally $\mathfrak{R}_{u}$ will be the subcollection of $\mathfrak{R}$ such that each $\& \in \mathfrak{R}_{u}$ has the property that limits of $\mathscr{L}$-convergent sequences are unique; $\mathfrak{R}_{u}^{*}$ is defined analogously.

Given a sequence $S$ and a point $x$ in $X$, let a class of sets $\beta$ in $X_{L}=S_{J} \cup\{x\}$ be defined as follows: (i) $0 \in B$; (ii) for all $x^{\prime} \in X_{L}$, if $x^{\prime} \neq x$ then $\left\{x^{\prime}\right\} \in \beta$; and (iii) for all non-negative $n, X_{n}=\left\{S_{j}: n<j\right\} \cup\{x\} \in \Theta$. Then $B$ is a base of open sets for a topology $\Im_{L}$ on $X_{L}$; this topology is compact and second countable, and is therefore compact metric if and only if it is Hausdorff. Viewing $S$ as converging to $x$ according to some rule, we shall call $J_{L}$ the sequence topology for the set $X_{L}$. Given a convergence class $\mathfrak{L} \in \mathbb{R}$, we accordingly can construct a collection of compact, second countable spaces 
$\left\{\left(X_{L}, J_{L}\right): L \in \mathfrak{L}\right\}$ covering $X$ by setting $X_{L}=S_{J} \cup\{x\}$ for every sequence $S$ and point $x$ in $X$ such that $S$ converges (\&) to $x$ and letting $J_{L}$ be the corresponding sequence topology on $X_{L}$.

2.1. Theorem. Let $\mathfrak{L} \in \mathfrak{R}$ and let $\left\{\left(X_{L}, \mathfrak{J}_{L}\right): L \in \mathfrak{L}\right\}$ be the associated collection of compact, second countable spaces. Then the following statements hold:

(a) $\left\{\left(X_{L}, J_{L}\right): L \in \mathcal{L}\right\}$ is a mosaic of compact metric spaces if and only if $\mathfrak{L} \in \mathfrak{R}_{u}$;

(b) if $\mathfrak{L} \in \mathfrak{R}_{u}$, then $\Psi(\mathfrak{L})=\mathfrak{J}$ and $\Phi(\mathfrak{J})=\mathfrak{L}^{*}$, where $\mathfrak{J}$ is the mosaic topology derived from $\left.\left\{X_{L}, \Im_{L}\right): L \in \mathfrak{L}\right\}$.

Proof. (a). Assume that $\mathscr{L} \in \mathcal{R}_{u}$, and endow $X_{L}=S_{J} \cup\{x\}$ with the sequence topology $\Im_{L}$, where $S$ converges $(\&)$ to $x$. For $x_{1}, x_{2} \in X_{L}$ if $x_{1} \neq x_{2}$, $x_{1} \neq x$, and $x_{2} \neq x$ then clearly $x_{1}$ and $x_{2}$ are separated by disjoint $J_{L}$-open sets. If $x_{1}=x$ and $x_{2} \neq x$ then there must exist an $X_{n}$ of the base $B$ of $J_{L}$ which contains $x_{1}=x$ but which does not contain $x_{2}$, for otherwise we could choose a subsequence of $S$ each of whose terms equals $x_{2}$; this subsequence would L-converge to both $x_{1}$ and $x_{2}$, contrary to the hypothesis of uniqueness of limits. Thus $\left\{x_{2}\right\}$ and $X_{n}$ are disjoint $J_{L}$-open sets separating $x_{1}$ and $x_{2}$. Since $\left(X_{L}, J_{L}\right)$ is Hausdorff it is compact metric.

Let $S$ converge $(\mathfrak{L})$ to $x$ and $T$ converge (\&) to $y$. Endow $X_{L}=S_{J} \cup\{x\}$ and $X_{M}=T_{J} \cup\{y\}$ with their respective sequence topologies $\Im_{L}$ and $\Im_{M}$. Assume that $E \subset X_{L}$ is $\Im_{L}$-closed. If $E \cap X_{M}$ is finite it is trivially $\Im_{M}$-closed; if $E \cap X_{M}$ is infinite it is sufficient to show that $y \in E \cap X_{M}$ in order to prove that it is $J_{M}$-closed. Assuming that $E \cap X_{M}$ is infinite let $R$ be a subsequence of $T$ such that $R_{J} \subset E \cap X_{M}, x \in R_{J}$, and $R$ has no constant subsequence. Then $R_{J} \subset S_{J}$, and consequently a sequence $P$ may be constructed such that $P<R$ and $P<S$. Therefore $P$ converges $(\mathscr{L})$ to $x$ and to $y$, and so $x=y$ by the hypothesis that $\mathscr{L} \in \mathfrak{R}_{u}$. But $E$ is infinite since $E \cap X_{M}$ is infinite, and so $E \in \Im_{L}$ implies that $x \in E$. Hence $y \in E \cap X_{M}$, and the compatibility condition holds. We conclude that $\left\{\left(X_{L}, J_{L}\right): L \in \mathscr{L}\right\}$ is a mosaic of compact metric spaces.

Conversely, assume that $\left\{\left(X_{L}, \Im_{L}\right): L \in \mathscr{L}\right\}$ is a mosaic of compact metric spaces. If $S$ converges $(\mathscr{L})$ to $x$ then it clearly converges $\left(\Phi\left(J_{L}\right)\right)$ to $x$, where $X_{L}=S_{J} \cup\{x\}$. Then $S$ converges $(\Phi(J))$ to $x$ by (1.2), where $J$ is the mosaic topology. Since $\mathfrak{L} \subset \Phi(\mathfrak{J})$ we have $\mathscr{L} \in \mathfrak{R}_{u}$ by (1.6).

(b). Assume that either one, and so both, of the conditions in (a) holds. Since $\mathscr{L} \subset \Phi(\mathfrak{J})$ it can be shown [11, Chapter XI] that $\Psi \Phi(\mathfrak{J}) \subset \Psi(\mathfrak{L})$. But the mosaic topology $J$ is full by (1.3), which can be shown to be equivalent to $J=\Psi \Phi(J)$; thus $J \subset \Psi(\mathfrak{L})$. On the other hand, if $E \notin J$ then by the definition of the mosaic topology $\mathfrak{J}$ there exists an $\left(X_{L}, J_{L}\right)$ such that $E \cap X_{L} \notin J_{L}$. Consequently there exists a sequence $T$ such that $T_{J} \subset E \cap X_{L}, T$ converges (\&) to $x$ where $X_{L}=S_{J} \cup\{x\}$, and $x \notin E \cap X_{L}$. Therefore $E \notin \Psi(\mathscr{L})$, and we conclude that $\Psi(\mathbb{L}) \subset \Im$. 
Now assume that $S$ converges $\left(\mathfrak{L}^{*}\right)$ to $x$, so that by the definition of *-extension every subsequence $S^{\prime}$ of $S$ has a subsequence $S^{\prime \prime}$ which converges

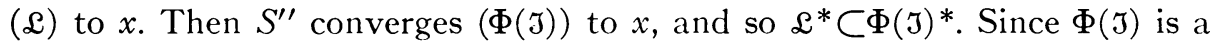
convergence class derived from a topology it is its own *-extension, and so $\mathfrak{L}^{*} \subset \Phi(\mathcal{J})$. Conversely, assume that $S$ does not converge $\left(\mathfrak{L}^{*}\right)$ to $x$, so that there exists a subsequence $S^{\prime}$ of $S$ such that no subsequence $S^{\prime \prime}$ of $S^{\prime}$ converges $(\mathfrak{L})$ to $x$. Then $S^{\prime}$ cannot converge $(\Phi(J))$ to $x$, for otherwise by an application of (1.5) we could find a subsequence $S^{\prime \prime}$ of $S^{\prime}$ which converges $\left(\Phi\left(J_{L}\right)\right)$ to $x$ for some $L \in \mathcal{L}$, which would yield contrary to hypothesis that $S^{\prime \prime}$ converges (L) to $x$. Since $S$ has the subsequence $S^{\prime}$ which does not converge $(\Phi(J))$ to $x$, it follows that $S$ does not converge $(\Phi(\mathfrak{J}))$ to $x$. Hence $\Phi(\mathfrak{J}) \subset \mathcal{L}^{*}$.

2.2. Corollary. Let $\mathfrak{L} \in \mathbb{R}$, then $\&$ coincides with the convergence class $\Phi(J)$ of a mosaic topology $\mathfrak{J}$ if and only if $\mathcal{L} \in \mathbb{R}_{u}^{*}$.

Proof. If $\mathfrak{L}=\Phi(\mathfrak{J})$ for some mosaic topology $\mathfrak{J}$ on $X$ then $\mathfrak{L} \in \mathbb{R}^{*}$ since it is a convergence class derived from a topology; by (1.6) limits are unique, and so $\mathscr{L} \in \mathfrak{R}_{u}^{*}$. Conversely, assume that $\mathfrak{L} \in \mathfrak{R}_{u}^{*}$, then $\mathfrak{L} \in \mathfrak{R}_{u}$ and $\mathfrak{L}=\mathfrak{L}^{*}$. By $(2.1)$ we find that $\Phi(\mathfrak{J})=\mathfrak{L}$, where $\mathfrak{J}$ is the mosaic topology of that theorem.

Now observe that the mapping $\mathfrak{I} \rightarrow \Phi(\mathfrak{J})$ carries the collection of mosaic topologies on a fixed set $X$ onto the collection $\mathfrak{\imath}_{u}^{*}$ of convergence classes, by virtue of (1.6) (2.2), and the fact that a convergence class derived from a topology is its own *-extension. In addition, if $J$ and $J^{\prime}$ are mosaic topologies such that $\Phi(J)=\Phi\left(J^{\prime}\right)$, then $\Psi \Phi(J)=\Psi \Phi\left(J^{\prime}\right)$, which implies that $J=J^{\prime}$ by (1.3). We conclude that the mosaic topologies on $X$ are precisely those topologies which are in one-to-one correspondence with the convergence classes of $\Omega_{u}^{*}$. A very useful means is now given for determining when a topological space is a mosaic space, by showing that the necessary conditions (1.3) and (1.6) are also sufficient.

2.3. Corollary. Let $(X, J)$ be a topological space such that $\mathfrak{J}$ is full and limits of $\Phi(J)$-convergent sequences are unique. Then $(X, J)$ is a mosaic space.

Proof. Since $\Phi(J) \in \mathfrak{R}_{u}$ we may construct a mosaic of compact metric spaces and the ensuing mosaic topology $J^{\prime}$, in the manner of $(2.1)$, wherein $\Psi \Phi(\mathfrak{J})=\mathfrak{J}^{\prime}$. Since $\mathfrak{J}$ is full we have $\mathfrak{J}=\Psi \Phi(\mathfrak{J})=\mathfrak{J}^{\prime}$.

By observing that a first countable space is Hausdorff if convergent sequences have unique limits, the following connection between mosaic spaces and first countable Hausdorff spaces is easily demonstrated.

2.4. Corollary. Let $(X, J)$ be a first countable topological space, then it is a mosaic space if and only if it is Hausdorff.

If a space $(X, J)$ possesses either the property that every $J$-countably compact set is $J$-closed or the property that every $\Phi(J)$-sequentially compact 
set is J-closed, then it is easily established that limits of $\Phi(\mathfrak{J})$-convergent sequences are unique. So either of these two properties together with the property that the topology $J$ is full characterizes a mosaic space. Any combination of the following properties which does not include the conditions of (2.3) or the two above mentioned sets of conditions need not imply that the space in question is a mosaic space:

(i) the topology $J$ is full;

(ii) limits of $\Phi(J)$-convergent sequences are unique;

(iii) for all $E \subset X, E$ is J-countably compact if and only if it is $\Phi(\mathfrak{J})$ sequentially compact;

(iv) for all $E \subset X$, if $E$ is $\Phi(J)$-sequentially compact then it is J-closed;

(v) for all $E \subset X$, if $E$ is J-countably compact then it is J-closed.

The following two examples indicate this.

2.5. Example. Let $X$ be the union of a sequence of plane subsets $X_{0}$, $X_{1}, \cdots$, where $X_{0}=\{(x, y): x=0 ; 0 \leqq y<1\}$ and $X_{n}=\{(x, y): x=1 / n$; $0<y<1\}$ for $n=1,2, \cdots$. Let the topology $\Im$ on $X$ be described by the following base of open sets: (i) for every $n=1,2, \cdots$, every point in $X_{n}$ has a neighborhood base consisting of all open subintervals of $X_{n}$ which contain the point; (ii) for a point in $X_{0}$ which is not equal to $(0,0)$ the base is all open subintervals of $X_{0}$ which contain the point; and (iii) for the point $(0,0)$ in $X_{0}$ the base is all sets of the form $Y_{0} \cup \cup\left\{Y_{m}: m=n, n+1, \cdots\right\}$ where $Y_{0}=\left\{(x, y): x=0 ; 0 \leqq y<b_{0}\right\}$ for $0<b_{0} \leqq 1$ and $Y_{m}=\{(x, y): x=1 / m$; $\left.0<y<b_{m}\right\}$ for $0<b_{m} \leqq 1$. Then this topology $\mathfrak{I}$ with its derived convergence class $\Phi(J)$ satisfies properties (ii) through (v) above, but not property (i). In fact, the full topology corresponding to the convergence class $\Phi(J)$ is that with the usual base of subintervals about all points including $(0,0)$.

2.6. Example. Let $X$ consist of the sequence of distinct points $\left\{x_{1}, x_{2}, \cdots\right\}$ and $y_{1}$ and $y_{2}$. Let an open neighborhood base for each $x_{n}$ be $\left\{x_{n}\right\}$, and for $y_{i}$, $i=1,2$, let the base consist of all sets of the form $\left\{y_{i}\right\} \cup\left\{x_{m}: m=n, n+1, \cdots\right\}$. For this space properties (i) and (iii) above hold, but not properties (ii), (iv), and $(\mathrm{v})$.

3. Functional mosaics. We now turn to a study of mosaics which entails functions.

3.1. Definition. Let the collection of triplets $\left\{\left(Y_{a}, \S_{a}, f_{a}\right): a \in A\right\}$ satisfy the following two conditions: (i) each $\left(Y_{a}, S_{a}\right)$ is a topological space; and (ii) each $f_{a}$ is a function defined on $Y_{a}$. Such a collection is said to be a functional mosaic whenever the following compatibility condition holds: for every $a, b \in A$, if $E \in S_{a}$ then $f_{b}^{-1} f_{a}(E) \in \delta_{b}$.

Such a structure has been previously employed in the study of compact Hausdorff varieties [4]. If each $f_{a}$ is one-to-one and each $Y_{a}$ is identified with $f_{a}\left(Y_{a}\right)$ then the functional mosaic reduces to a mosaic of topological spaces. Whenever the collection reduces to one triplet $(Y, s, f)$ compatibility becomes the following: for all $E \subset Y$, if $E \in S$ then $f^{-1} f(E) \in \mathcal{S}$. In this case $f$ is said to be 
self-compatible for the topology $s$. We remark that a decomposition $D$ of a topological space $(Y, S)$ is upper semi-continuous if and only if the projection function $f: Y \rightarrow D$ is self-compatible.

For any such collection $\left\{\left(Y_{a}, \S_{a}, f_{a}\right): a \in A\right\}$, compatible or otherwise, a topology $J$ may be defined on its range set $X=\bigcup\left\{f_{a}\left(Y_{a}\right): a \in A\right\}$ in the following standard manner: for all $E \subset X, E \in J$ if and only if $f_{a}^{-1}(E) \in \mathcal{S}_{a}$ for all $a \in A$. Then $\Im$ is the finest topology on $X$ for which each function $f_{a}$ is continuous. A collection of topological spaces $\left\{\left(X_{a}, J_{a}\right): a \in A\right\}$ covering $X$ is obtained as follows: for all $a \in A, X_{a}=f_{a}\left(Y_{a}\right)$ and for all $E \subset X_{a}, E \in J_{a}$ if and only if $f_{a}^{-1}(E) \in S_{a}$. An expanded collection of topological spaces $\left\{\left(X_{b}, J_{b}\right): b \in B\right\}$ covering $X$ is similarly obtained. Let $B$ consist of all ordered pairs $(a, E)$ such that $a \in A$ and $E \in \mathcal{S}_{a}$. For $b=(a, E) \in B$ define $X_{b}=f_{a}(E)$, and define the topology $\Im_{b}$ on $X_{b}$ by demanding, for all $F \subset X_{b}$, that $F \in J_{b}$ if and only if $f_{a}^{-1}(F) \in S_{a}$. In particular, if $b=\left(a, Y_{a}\right)$ for some $a \in A$ then $X_{b}=X_{a}$ and $J_{b}=J_{a}$. Another topology $J^{\prime}$ may be defined on $X$ by taking as a sub-base for the closed sets the collection $\left\{f_{a}(E): a \in A ; E \in \mathcal{S}_{a}\right\}$. The topology $J^{\prime}$ is the coarsest topology on $X$ for which each $f_{a}$ is a closed function. Implicit in the definitions introduced is the first lemma.

3.2. Lemma. For the structures defined above the following statements hold:

(a) for all $E \subset X, E \in J$ if and only if $E \cap X_{a} \in J_{a}$ for all $a \in A$;

(b) for all $E \subset X, a \in A$, if $E \in J_{a}$ then $E \in J^{\prime}$;

(c) for all $E \subset X, a \in A$, if $E \subset X_{a}$ and $E \in J$ then $E \in J_{a}$;

(d) for all $E \subset X, b \in B$, if $E \subset X_{b}$ and $E \in J$ then $E \in J_{b}$.

The first theorem gives several necessary and sufficient conditions for a collection of triplets to be a functional mosaic.

3.3. Theorem. Let $\left\{\left(Y_{a}, S_{a}, f_{a}\right): a \in A\right\}$ be a collection of triplets satisfying the conditions (i) and (ii) of (3.1). Then the following statements are equivalent:

(a) each function $f_{a}$ is closed and continuous for the topology $\mathrm{J}$;

(b) $\left\{\left(Y_{a}, S_{a}, f_{a}\right): a \in A\right\}$ is a functional mosaic;

(c) $J$ is finer than $J^{\prime}$;

(d) for all $a \in A, J$ and $J^{\prime}$ relativized to $X_{a}$ coincide with $\Im_{a}$;

(e) each function $f_{a}$ is closed and continuous for the topology $J^{\prime}$;

(f) for the covering $\left\{\left(X_{b}, J_{b}\right): b \in B\right\}$ of $X, X_{b} \cap X_{b^{\prime}} \in J_{b^{\prime}}$ for all $b, b^{\prime} \in B$;

(g) each function $f_{a}$ is self-compatible for the topology $S_{a}$, and $\left\{\left(X_{a}, J_{a}\right)\right.$ : $a \in A\}$ is a mosaic of topological spaces.

Proof. (a) $\rightarrow$ (b). Let $E \in S_{a}$, then $f_{a}(E) \in J$ by (a), and so $f_{a^{\prime}}^{-1} f_{a}(E) \in S_{a^{\prime}}$ by the continuity of $f_{a^{\prime}}$.

(b) $\rightarrow$ (c). Let $E \in \mathcal{S}_{a}$ so that $f_{a}(E)$ is a typical element of the sub-base for $J^{\prime}$. Then $f_{a^{\prime}}^{-1} f_{a}(E) \in \delta_{a^{\prime}}$ for all $a^{\prime} \in A$. Therefore $f_{a}(E) \in J$ by definition.

$(\mathrm{c}) \rightarrow(\mathrm{d})$. Let $E \in J_{a}$, then $E \in J^{\prime}$ by (3.2.b), and so $E \in J$ by (c), which implies $E \in J_{a}$ by (3.2.c). 
$(\mathrm{d}) \rightarrow(\mathrm{e})$. It is sufficient to show that each $f_{a}$ is $\mathrm{J}^{\prime}$-continuous. Let $E \in S_{a^{\prime}}$ so that $f_{a^{\prime}}(E)$ is a typical element of the sub-base for $\mathfrak{J}^{\prime}$. Then $f_{a^{\prime}}(E) \cap f_{a}\left(Y_{a}\right)$ $=f_{a^{\prime}}(E) \cap X_{a} \in J^{\prime}$. By (d) the topologies $J$ and $J^{\prime}$ coincide on $X_{a}$, hence $f_{a}^{-1}\left[f_{a^{\prime}}(E) \cap X_{a}\right]=f_{a}^{-1} f_{a^{\prime}}(E) \in S_{a}$ by the J-continuity of $f_{a}$. Hence each $f_{a}$ is $J^{\prime}$-continuous.

(e) $\rightarrow$ (a). It is sufficient to show that each $f_{a}$ is J-closed. Let $E \in S_{a}$, then $f_{a}(E) \in J^{\prime}$ by definition, and so $f_{a^{\prime}}^{-1} f_{a}(E) \in S_{a^{\prime}}$ by $(\mathrm{e})$. Thus $f_{a}(E) \in J$ by definition, and each $f_{a}$ is 3 -closed.

(c) $\rightarrow$ (f). By definition $X_{b}$ and $X_{b^{\prime}}$ are $J^{\prime}$-closed, and therefore 5 -closed by (c). Hence $X_{b} \cap X_{b^{\prime}} \in J$, and so $X_{b} \cap X_{b^{\prime}} \in J_{b^{\prime}}$ by (3.2.d).

(f) $\rightarrow$ (c). Let $E$ be an element of the sub-base for $J^{\prime}$, then $E=X_{b}$ for some $b \in B$. Hence $E \cap X_{a} \in J_{a}$ for all $a \in A$ by (f), implying $E \in J$ by (3.2.a).

(b) $\rightarrow(\mathrm{g})$. If (b) holds then obviously each function $f_{a}$ is self-compatible. Now (b) is equivalent to (f), so if $E \in J_{a}$ then $E=X_{b}$ for $b=\left(a, f_{a}^{-1}(E)\right)$ and $X_{a^{\prime}}=X_{b^{\prime}}$ for $b^{\prime}=\left(a^{\prime}, Y_{a^{\prime}}\right)$. Hence $E \cap X_{a^{\prime}}=X_{b} \cap X_{b^{\prime}} \in J_{b^{\prime}}=J_{a^{\prime}}$.

$(\mathrm{g}) \rightarrow(\mathrm{b})$. If $E \in \mathcal{S}_{a}$ then $f_{a}(E) \in J_{a}$ since $f_{a}$ is assumed to be self-compatible. Then $f_{a}(E) \cap X_{a^{\prime}} \in J_{a^{\prime}}$, and so $f_{a^{\prime}}^{-1}\left[f_{a}(E) \cap X_{a^{\prime}}\right]=f_{a^{\prime}}^{-1} f_{a}(E) \in \mathcal{S}_{a^{\prime}}$.

If the collection of triplets is specialized to one triplet the following corollary is immediate.

3.4. Corollary. Let $f$ be a function from the topological space $(Y, s)$ onto the set $X$. Then the following conditions are equivalent:

(a) the function $f$ is self-compatible for the topology $s$;

(b) for the topologies $\Im$ and $\mathfrak{J}^{\prime}$ defined above, $\Im=J^{\prime}$;

(c) the collection of triplets $\left\{\left(E, \mathfrak{S}_{E}, f_{E}\right): E \in \mathcal{S}\right\}$, where $\mathfrak{S}_{E}$ is $\mathcal{S}$ relativized to the $\delta$-closed set $E$ and $f_{E}$ is $f$ restricted to $E$, is a functional mosaic.

Heretofore in constructing a mosaic space we have used a mosaic of compact metric spaces. The next theorem shows that there is considerable freedom in the construction of such a space, for either a mosaic of topological spaces or a functional mosaic may be employed, in which the topological spaces of the mosaic are metric, first countable Hausdorff, etc., or ultimately merely mosaic spaces.

3.5. Theorem. Let $\left\{\left(Y_{a}, S_{a}, f_{a}\right): a \in A\right\}$ be a functional mosaic, for which each $\left(Y_{a}, \mathrm{~S}_{a}\right)$ is a mosaic space. Let $\mathrm{I}$ be the finest topology on the range set $X=\bigcup\left\{f_{a}\left(Y_{a}\right): a \in A\right\}$ for which each function $f_{a}$ is continuous. Then $(X, J)$ is a mosaic space.

Proof. For each $a \in A$ let $\left\{\left(Y_{a p}, \delta_{a p}\right): p \in P_{a}\right\}$ be a mosaic of compact metric spaces on $Y_{a}$ which determines the topology $S_{a}$. For each $a \in A, p \in P_{a}$, let $X_{a p}=f_{a}\left(Y_{a p}\right)$ and define the topology $\mathcal{J}_{a p}$ on $X_{a p}$ by demanding, for all $F \subset X_{a p}$, that $F \in J_{a p}$ if and only if $f_{a}^{-1}(F) \in \mathcal{S}_{a}$. Since each $Y_{a p}$ is an $\S_{a}$-closed subsent of $Y_{a}$ it follows that $\left\{\left(X_{n_{p}}, T_{\sigma_{p}}\right): a \in A ; p \in P_{a_{b}}\right\}$ is a subcollection of the covering $\left\{\left(X_{b}, J_{i}\right): b \in B\right\}$ which has been previously introduced. From 
condition (f) of (3.3) it follows that $\left\{\left(X_{a p}, \Im_{a p}\right): a \in A ; p \in P_{a}\right\}$ is a mosaic of topological spaces on $X$. Since each $f_{a}$ is self-compatible for the topology $\mathcal{S}_{a}$ it follows that $\left(X_{a p}, J_{a p}\right)$ is the closed, continuous image of the compact metric space $\left(Y_{a p}, \S_{a p}\right)$ under $f_{a p}$, where $f_{a p}$ is $f_{a}$ restricted to $Y_{a p}$. Therefore each $\left(X_{a p}, J_{a p}\right)$ is a compact metric space.

From the mosaic of compact metric spaces $\left\{\left(X_{a p}, J_{a p}\right): a \in A ; p \in P_{a}\right\}$ there is obtained the associated mosaic topology $\Im_{0}$ in the usual way: for all $E \subset X, E \in J_{0}$ if and only if $E \cap X_{a p} \in J_{a p}$ for all $a \in A, p \in P_{a}$. The proof is completed by showing that $\Im_{0}=\Im$. Now $E \in J$ if and only if $f_{a}^{-1}(E) \in \mathcal{S}_{a}$ for all $a \in A$. Since for each fixed $a \in A$ the mosaic of compact metric spaces $\left\{\left(Y_{a p}, \varsigma_{a p}\right): p \in P_{a}\right\}$ determines the topology $\S_{a}$ on $Y_{a}$ it follows that $E \in \mathcal{J}$ is equivalent to $f_{a}^{-1}(E) \cap Y_{a p}=f_{a p}^{-1}\left(E \cap X_{a p}\right) \in \mathcal{S}_{a p}$ for all $a \in A, p \in P_{a}$. But a subset $F$ of $X_{a p}$ is $\Im_{a p}$-closed if and only if $f_{a p}^{-1}(F) \in \mathcal{S}_{a p}$. Therefore $E \in J$ if and only if $E \cap X_{a p} \in J_{a p}$ for all $a \in A, p \in P_{a}$, and so $\Im_{0}=\Im$.

By specializing to one triplet $(Y, S, f)$ and observing that then the conditions of the above theorem are that $f$ be closed and continuous for the topology $\mathfrak{J}$ on $f(Y)$, the following corollary results.

3.6. Corollary. Let $f$ be a closed, continuous function from the mosaic space $(Y, \delta)$ onto the topological space $(X, J)$. Then $(X, J)$ is a mosaic space.

By relaxing the properties of $f$ from closed and continuous to quasicompact, namely $E \in J$ if and only if $f^{-1}(E) \in \mathcal{S}$, and adding a "separation" property to the topology of the range space we again get the mosaic property to carry over.

3.7. Theorem. Let $f$ be a quasi-compact function from the mosaic space $(Y, \S)$ onto the topological space $(X, J)$ for which it is assumed that $\Phi(J)$ convergent sequences have unique limits. Then $(X, J)$ is a mosaic space.

Proof. By (2.3) it is sufficient to show that the topology $J$ is full. Assume that $E \subset X$ is not J-closed, then $f^{-1}(E) \notin S$ since $f$ is quasi-compact. Since $\delta$ is full by (1.3) there exists a sequence $S$ such that $S_{J} \subset f^{-1}(E)$ and $S$ converges $(\Phi(S))$ to $y \in f^{-1}(E)$. By the continuity of $f$ the sequence $f S$ converges $(\Phi(\Im))$ to $f(y)$; in addition $f S_{J} \subset E$ and $f(y) \notin E$. We conclude that $J$ is full.

Next, a useful converse to the above theorem is given.

3.8. Theorem. Every mosaic space is the quasi-compact image of a suitable locally compact metric space.

Proof. Let $(X, J)$ be a mosaic space whose topology is determined by the mosaic of compact metric spaces $\left\{\left(X_{a}, \Im_{a}\right): a \in A\right\}$. Let $M=\{(a, x): a \in A$; $\left.x \in X_{a}\right\}$, observe that $M=\bigcup\left\{M_{a}: a \in A\right\}$ where the $M_{a}$-sets are disjoint copies of the $X_{a}$-sets. Define $f: M \rightarrow X$ by demanding that $f(a, x)=x$ for all $(a, x) \in M$. Provide $M$ with a topology such that $f$ restricted to $M_{a}$ is a homeomorphism onto $X_{a}$, for all $a \in A$, and each set $M_{a}$ is both open and closed in the topology. 
Then it is easily seen that $M$ is a locally compact metric space, and that $f$ is quasi-compact. (Compare this with Cohen's result for $k$-spaces [3, Theorem 1.8].)

3.9. Example. Recently Stone has shown [10, Theorem $\left.3^{\prime}\right]$ that if $f: M \rightarrow X$ is a quasi-compact function from the locally compact, metric space $M$ onto the first countable, paracompact, Hausdorff space $X$ then $X$ is locally compact and metric, provided the frontier of each set of the form $f^{-1}(x)$ is separable, and then raises the question as to whether the frontier hypothesis is superfluous. We now indicate that this is not the case. Let $X$ be any space which is first countable, paracompact, and Hausdorff, but which is not metric. By (2.4) it is a mosaic space, and so by (3.8) it is the quasicompact image of a locally compact, metric space.

4. Hereditary mosaic spaces. A metric space, for example, has the hereditary property that each subspace is a metric space. By analogy the question arises as to whether a mosaic space is hereditary in the sense that each subspace is a mosaic space, for which the answer is in general negative. The following material centers around this consideration.

4.1. Theorem. If $Y$ is a $\mathfrak{J}$-open or $\mathfrak{J}$-closed subset of a mosaic space $(X, \mathfrak{J})$ with the relative topology $s$ then $(Y, \delta)$ is a mosaic space.

Proof. Since $\Phi(J)$-convergent sequences have unique limits by (1.6) it easily follows that $\Phi(S)$-convergent sequences have unique limits. By (2.3) the proof is complete once it is shown that $S$ is full.

Assume that $Y \in \mathcal{J}$, and for $E \subset Y$ assume that $E \in \Psi \Phi(\mathcal{S})$. Let $S$ be a sequence such that $S_{J} \subset E$ and $S$ converges $(\Phi(J))$ to $x$. Since $Y \in J$ and $E \subset Y$ we have $x \in Y$, and so $S$ converges $(\Phi(S))$ to $x$. But it can be shown that $\Phi(\delta)=\Phi \Psi \Phi(\delta)$, and so $S$ converges $\Phi \Psi \Phi(\delta)$ to $x$, implying that $x \in E$ since $E \in \Psi \Phi(\mathcal{S})$. Thus $E \in \Psi \Phi(J)$, and so $E \in J$ by (1.3). We conclude that $E \in S$, and so $S$ is full.

Alternatively, assume that $Y$ is J-open and for $E \subset Y$ assume that $E$ is $\Psi \Phi(S)$-open. Let $S$ be a sequence which converges $(\Phi(J))$ to $x \in E$. Since $Y$ is J-open the sequence $S$ is eventually in $Y$, and so it converges $(\Phi(S))$ to $x$. By the assumption that $E$ is $\Psi \Phi(S)$-open the sequence $S$ is eventually in $E$. Therefore $E$ is $\Psi \Phi(J)$-open, and so J-open by (1.3). We conclude that $E$ is $\mathcal{S}$-open, and so $\mathcal{S}$ is full.

Next, we give an example of a mosaic space in which not every subspace is a mosaic space.

4.2. Example. Let $X$ be the subset of the plane consisting of the union of arcs, $X=\mathrm{U}\left\{X_{n}: n=0,1,2, \cdots\right\}$, where $X_{0}=\{(x, y): 0 \leqq x \leqq 1 ; y=0\}$ and for $n=1,2, \cdots, X_{n}=\{(x, y): x=1 / n ; 0 \leqq y \leqq 1\}$. Take this collection of $\operatorname{arcs}\left\{X_{n}: n=0,1,2, \cdots\right\}$ with their usual metric topologies as a mosaic of compact metric spaces on $X$ and generate accordingly the associated mosaic topology $J$. Then the point $(0,0)$ is a $J$-accumulation point of the set $Y$ 
$=\bigcup\left\{Y_{n}: n=1,2, \cdots\right\}$ where $Y_{n}=\{(x, y): x=1 / n ; 0<y \leqq 1\}$, but no sequence in $Y$ converges $(\Phi(J))$ to $(0,0)$. Consequently the set $Y^{\prime}=\{(0.0)\} \cup Y$ with its relative topology $\delta$ is not a mosaic space, since $\{(0,0)\}$ is not $S$-open but is open for the full-refinement $\Psi \Phi(\delta)$.

Clearly in any mosaic space the class of mosaic subspaces is partially ordered by set inclusion. Nevertheless, no decent lattice properties need obtain, as this example demonstrates. $Y$ and $\{(0,0)\}$ are mosaic subspaces but their union $Y^{\prime}$ is not a mosaic subspace. Let $Z^{\prime}=\bigcup\left\{Z_{n}: n=1,3, \cdots\right\}$ $\cup Y^{\prime}$ and $Z^{\prime \prime}=\bigcup\left\{Z_{n}: n=2,4, \cdots\right\} \cup Y^{\prime}$, where $Z_{n}=\{(x, y): 1 /(n+1)<x$ $<1 / n ; y=0\}$ for $n=1,2, \cdots$. Then $Z^{\prime}$ and $Z^{\prime \prime}$ are mosaic subspaces but $Z^{\prime} \cap Z^{\prime \prime}=Y^{\prime}$ is not a mosaic subspace. Let $Z=\{(x, y): 0<x \leqq 1 ; y=0\}$. Then $Z$ is a mosaic subspace but $X-Z=Y^{\prime}$ is not a mosaic subspace.

Pursuing the counterexample further, we now indicate that $(X, J)$ is the image of the half-line $[0, \infty)$ with the usual metric topology under a quasicompact function that is defined as follows: (i) $f(x)=(x, 0)$ for $0 \leqq x \leqq 1$; (ii) $f(x)=(1 / n, x-3 n+2)$ for $3 n-2 \leqq x \leqq 3 n-1$; (iii) $f(x)=(1 / n, 3 n-x)$ for $3 n-1 \leqq x \leqq 3 n$; and (iv) $f(x)=\left([4 n+1-x] /\left[n^{2}+n\right], 0\right)$ for $3 n \leqq x \leqq 3 n+1$. In order to prove that $f$ is quasi-compact we must show that $f$ is continuous and that for all $E \subset X$ if $E \notin J$ then $f^{-1}(E)$ is not closed for the metric topology on $[0, \infty)$. But continuity is obvious, and if $E \notin J$ then there exists an arc $X_{n}$ of the mosaic such that $E \cap X_{n}$ is not closed for this arc's topology. Then clearly $f^{-1}(E)$ is not closed for the metric topology of $[0, \infty)$.

The situation under which this subspace difficulty disappears is expressed by the next theorem.

4.3. ThEOREM. Let $(X, J)$ be a mosaic space, then it is hereditary if and only if the following statement holds: for every set $E$ and point $x$ in $X$ if $x$ is a J-accumulation point of $E$ then there exists a sequence $S$ such that $S_{J} \subset E$ and $S$ converges $(\Phi(\mathfrak{J}))$ to $x$.

Proof. Assume that there exists a set $E$ and a point $x$ in $X$ such that $x$ is a J-accumulation point of $E$ but no sequence in $E$ converges $(\Phi(J))$ to $x$. On the set $E \cup\{x\}$ let $\delta$ be the relative topology, then $S$ converges $(\Phi(\delta))$ to $y$ if and only if it converges $(\Phi(J))$ to $y$, for all $S$ and $y$ in $E \cup\{x\}$. Now the set $\{x\}$ is not $S$-open as $x$ is an $S$-accumulation point of $E$, but it is open in the full-refinement $\Psi \Phi(\delta)$ of $\delta$ since the only sequences which $\Phi(\delta)$-converge to $x$ are those which are eventually in $\{x\}$. We conclude that $\delta$ is not full, and so $(E \cup\{x\}, \delta)$ is not a mosaic space by (1.3).

Conversely, assume for every set $E$ and point $x$ in $X$ that if $x$ is a $J$ accumulation point of $E$ then there exists a sequence $S$ such that $S_{J} \subset E$ and $S$ converges $(\Phi(J))$ to $x$. Let $(Y, \mathcal{S})$ be a subspace of $(X, \Im)$. Since uniqueness of limits carries over for the convergent sequences of a relative topology it is sufficient to show that $\delta$ is full in order to complete the proof by (2.3). For $E \subset Y$ assume that $E \in \Psi \Phi(S)$. Let $\bar{E}$ be the J-closure of $E$, then clearly 
$E \subset \bar{E} \cap Y$. Now suppose that there exists an $x \in(\bar{E} \cap Y)-E$. Since $x$ is a J-accumulation point of $E$ there exists by hypothesis a sequence $S$ such that $S_{J} \subset E$ and $S$ converges $(\Phi(J))$ to $x$. Since $x \in Y, E \subset Y$ it follows that $S$ converges $(\Phi(S))$ to $x$. Since $E \in \Psi \Phi(S)$ we have $x \in E$. Therefore a contradiction is reached, which implies that $\bar{E} \cap Y \subset E$. We conclude that $E \in \mathcal{S}$, and so $\mathcal{S}$ is full.

Under a restriction the hereditary property carries over for a functional mosaic, as the subsequent theorem shows.

4.4. Theorem. Let $\left\{\left(Y_{n}, \mathcal{S}_{n}, f_{n}\right): n=1, \cdots, N\right\}$ be a finite functional mosaic, for which each $\left(Y_{n}, S_{n}\right)$ is an hereditary mosaic space. Let $\mathrm{J}$ be the finest topology on $X=\bigcup\left\{f_{n}\left(Y_{n}\right): n=1, \cdots, N\right\}$ which makes each function $f_{n}$ continuous. Then $(X, \Im)$ is an hereditary mosaic space.

Proof. By (3.5) it suffices to prove the hereditary property. We may assume that the sets $Y_{n}$ are pairwise disjoint. Let $Y=\bigcup\left\{Y_{n}: n=1, \cdots, N\right\}$ and define a topology $\delta$ on $Y$ by demanding that each $Y_{n}$ be open and closed in $\delta$, and that $\mathcal{S}$ relativized to $Y_{n}$ be $\mathcal{S}_{n}$. Define $f: Y \rightarrow X$ by $f(y)=f_{n}(y)$ for $y \in Y_{n}$. Then $(Y, \S)$ is an hereditary mosaic space and $f$ is a closed, continuous function onto $X$. Thus the proof reduces to showing that the hereditary property carries over under a closed, continuous function.

For $E$ and $x$ in $X$ assume that $x$ is a J-accumulation point of $E$. For $f^{-1}(E)$ and $f^{-1}(x)$ in $Y$ assume for every $y \in f^{-1}(x)$ that there exists a set $F_{y} \subset Y$ such that $F_{y} \in \mathcal{S}, f^{-1}(E) \subset F_{y}$ and $y \notin F_{y}$. Then $F=\bigcap\left\{F_{y}: y \in f^{-1}(x)\right\}$ is $\mathcal{S}$-closed, $f^{-1}(E) \subset F$, and $f^{-1}(x) \cap F=0$. Therefore $f(F) \in J$ since $f$ is a closed function, $E \subset f(F)$, and $x \notin f(F)$. But this contradicts the assumption that $x$ is a Jaccumulation point of $E$, and so there exists a $y \in f^{-1}(x)$ such that for all $G \subset Y$, if $G \in \delta$ and $f^{-1}(E) \subset G$ then $y \in G$. Thus $y$ is an $\delta$-accumulation point of $f^{-1}(E)$, and since $(Y, \delta)$ is hereditary by (4.3) there exists a sequence $S$ such that $S_{J} \subset f^{-1}(E)$ and $S$ converges $(\Phi(S))$ to $y$. Since $f$ is continuous the sequence $f S$ converges $(\Phi(J))$ to $f(y)=x$. In addition, $f S_{J} \subset E$. By (4.3) the space $(X, J)$ is hereditary.

Since the example of (4.2) furnishes a nonhereditary mosaic space which is the quasi-compact image of an hereditary mosaic space it is in general not possible to carry over the hereditary property under a quasi-compact function. At the same time (4.2) yields a counterexample to the generalization of (4.4) to infinite functional mosaics, for the countable collection of functions $\left\{f_{n}: n=1,2, \cdots\right\}$, where each $f_{n}$ is $f$ of (4.2) restricted to the closed interval $[n-1, n]$, provides an infinite functional mosaic for which each domain space is an hereditary mosaic space, yet the range space is not hereditary.

5. Maximal mosaics. Let $X$ be a fixed set and let $\mathfrak{M}$ be the collection of all mosaics of compact metric spaces on $X$; the collection $\mathfrak{M}$ is clearly partially ordered by set inclusion. I et $P$ be a chain of masnics in $M$. Sinre the element $\mathfrak{r}^{\prime}=\bigcup\{\mathfrak{U}: \mathfrak{N} \in \mathfrak{R}\}$ satisfics the compatibility condition it is a mosaic 
of compact metric spaces which is an upper bound for the chain of mosaics $\mathfrak{N}$. By Zorn's lemma we conclude that there exists a maximal mosaic in $\mathfrak{M}$. Now let $\mathfrak{T}$ be such a maximal mosaic, and let $\mathfrak{T}_{f}$ be its full-extension, namely the mosaic of all compact metric subspaces of the mosaic space generated by $\mathfrak{T}$. Since $\mathscr{M}$ is maximal and $\mathscr{T} \subset \mathfrak{N}_{f}$ we have $\mathfrak{N}=\mathfrak{M}_{f}$. Therefore every maximal mosaic is full. Let $\mathfrak{R}_{u}$ be the collection of convergence classes previously defined, and partially ordered it by set inclusion. By reasoning analogous to the above there are maximal elements in $\mathfrak{R}_{u}$.

By observing according to (1.8) that in a mosaic space every countably compact set is closed we obtain the following result, whose easy proof will be omitted.

5.1. Lemma. Let $(X, J)$ be a mosic space and let $\delta$ be a topology on $X$ which is properly finer than $\Im$. Then $(X, \delta)$ is not countably compact.

5.2. Theorem. Let $(X, J)$ be a mosaic space, Th its full mosaic of compact metric subspaces, and $\Phi(J)$ its derived convergence class. Then the following statements are equivalent:

(a) the topology $\mathrm{J}$ is countably compact;

(b) the full mosaic $\mathfrak{M}$ is maximal in $\mathfrak{M}$;

(c) the convergence class $\Phi(\mathfrak{J})$ is maximal in $\mathfrak{R}_{u}$.

Proof. (a) $\leftrightarrow(b)$. Assume that $(X, J)$ is not countably compact. Since $(X, J)$ is a $T_{1}$-space there exists a countable subset $Y$ which has no J-accumulation point. Then by (1.4) the set $Y \cap X_{0}$ consists of at most a finite number of points, for every $\left(X_{0}, J_{0}\right) \in \mathfrak{T}$. By arbitrarily endowing $Y$ with a compact metric topology $\delta$ there is obtained a space $(Y, \delta)$ which is compatible with every $\left(X_{0}, J_{0}\right) \in \mathscr{M}$, and for which $(Y, s) \notin \mathscr{T}$. Therefore $\mathfrak{T}$ is not maximal.

Conversely, assume that the full mosaic $\mathscr{M}$ is not maximal so that there exists a compact metric space $(Y, \S)$ which is compatible with every element of $\mathfrak{M}$, but for which $(Y, \delta) \notin \mathfrak{T}$, so that $(Y, \delta)$ is not a subspace of $(X, \mathfrak{J})$. By compatibility, for all $E \subset Y$, if $E \in \mathcal{S}$ then $E \in J$. Therefore $J_{Y}$ is properly finer than $\mathcal{S}$, where $\mathfrak{J}_{Y}$ is $J_{\text {relativized to }} Y$. By $(5.1)$ the space $\left(Y, J_{Y}\right)$ is not countably compact, implying that $Y$ is not $J$-countably compact. Since $Y \in J$ we conclude that $(X, J)$ is not countably compact.

$(\mathrm{a}) \leftrightarrow(\mathrm{c})$. By (1.7) it is sufficient to show that $\Phi(\mathfrak{J})$ is sequentially compact if and only if (c) holds. Observe that $\Phi(\mathfrak{J}) \in \mathfrak{R}_{u}$ by (1.6). Assume that $\Phi(J)$ is sequentially compact and let $\mathscr{L}$ be a convergence class such that $\Phi(J) \subset \mathscr{L} \in \mathfrak{R}_{u}$. Now let the sequence $S$ converge (L) to $x$, and let $S^{\prime}<S$ so that $S^{\prime}$ converges $(\mathscr{L})$ to $x$. Since $\Phi(J)$ is sequentially compact there exists a sequence $S^{\prime \prime}$ such that $S^{\prime \prime} \prec S^{\prime}$ and $S^{\prime \prime}$ converges $(\Phi(J))$ to some $y$. Since $\Phi(\mathfrak{J}) \subset \mathfrak{L}$ we have that $S^{\prime \prime}$ converges $(\mathfrak{L})$ to $y$. On the other hand, $S^{\prime \prime}$ converges (L) to $x$ since $S^{\prime \prime}<S^{\prime}<S$, and so $x=y$ by $\mathscr{L} \in \mathfrak{R}_{u}$. Since every subsequence 
of $S$ has a subsequence which converges $(\Phi(J))$ to $x$ we conclude that $S$ converges $\left(\Phi(J)^{*}\right)$ to $x$. But $\Phi(J)=\Phi(J)^{*}$, and so $\mathscr{L} \subset \Phi(J)$. Therefore $\Phi(J)$ is maximal in $\mathfrak{R}_{u}$.

Conversely, assume that $\Phi(\mathfrak{J})$ is not sequentially compact so that there exists a sequence $S$ such that every subsequence converges $(\Phi(J))$ to no point in $X$. To this sequence and its subsequences arbitrarily assign a limit $x \in X$. Let $\mathscr{L}$ be the convergence class consisting of the $\Phi(J)$-elements to which have been added the subsequences of $S$ with the limit $x$. Then $\mathfrak{L} \in \mathcal{R}_{u}$, and in addition it is properly larger than $\Phi(J)$. Therefore $\Phi(J)$ is not maximal in $\mathfrak{R}_{u}$.

We now turn to the compactification problem. Let $(X, J)$ be any topological space; to $X$ add the point $x^{\prime}$ and on $X^{\prime}=X \cup\left\{x^{\prime}\right\}$ define a topology $J^{\prime}$ by demanding for all $E \subset X^{\prime}$ that $E$ is $\Im^{\prime}$-open if and only if either $E$ is J-open or $X^{\prime}-E$ is $J$-closed and $J$-countably compact. Then $\left(X^{\prime}, J^{\prime}\right)$ is a countably compact topological space, $(X, J)$ is an open subspace, and $\left(X^{\prime}, J^{\prime}\right)$ is Hausdorff only if $(X, J)$ is Hausdorff and locally countably compact. The justification of these statements will be omitted, as it is analogous to that of results for the Alexandroff compactification [7, Chapter 5].

5.3. Theorem. Let $(X, J)$ be a topological space and let $\left(X^{\prime}, J^{\prime}\right)$ be its one point countable compactification. Then $\left(X^{\prime}, \mathfrak{J}^{\prime}\right)$ is a mosaic space if and only if $(X, \mathfrak{J})$ is a mosaic space.

Proof. Assume that $\left(X^{\prime}, J^{\prime}\right)$ is a mosaic space. Since $(X, J)$ is an open subspace it follows from (4.1) that it is a mosaic space. Conversely, assume that $(X, J)$ is a mosaic space. Let $S$ converge $\left(\Phi\left(\Im^{\prime}\right)\right)$ to $x$ and to $y$. If $x, y \in X$ then $x=y$ by (1.6) and the fact that $(X, \Im)$ is an open subspace. If $y=x^{\prime}$ and $x \in X$ then $S_{J} \cup\{x\}$ is J-closed and J-countably compact. Thus $X^{\prime}-\left(S_{J} \cup\{x\}\right)$ is a $J^{\prime}$-open set containing $y$, and the $\Phi\left(J^{\prime}\right)$-convergence of $S$ to $y$ is denied. We conclude that limits of $\Phi\left(J^{\prime}\right)$-convergent sequences are unique. By (2.3) the proof is completed once it is shown that $\mathfrak{J}^{\prime}$ is full.

Let $E \subset X^{\prime}$ be a set which is not $J^{\prime}$-closed. If $x^{\prime} \in E$ then $X^{\prime}-E$ is not J-open, which implies by (1.3) that there is a sequence $S$ for which $S_{J} \subset E$ $-\left\{x^{\prime}\right\}$ and $S$ converges $(\Phi(J))$ to $x \in X^{\prime}-E$. Therefore $S_{J} \subset E$ and $S$ converges $\left(\Phi\left(J^{\prime}\right)\right)$ to $x \notin E$. Alternatively, if $E \subset X$ then $E$ is not J-countably compact by (1.8), and so there exists a countable set $F \subset E$ such that $F$ has no J-accumulation point in $E$. If $F$ has any $J$-accumulation point $y \notin E$, then clearly $E$ is not J-closed and we can again find a sequence $S$ in $E$ which converges $(\Phi(J))$, and so also converges $\left(\Phi\left(J^{\prime}\right)\right)$, to a point $x \in E$. If $F$ has no J-accumulation point let $S$ be a sequence such that $S_{j}=S_{k}$ if and only if $j=k$ and $S_{J}=F$. Then $S$ converges $\left(\Phi\left(J^{\prime}\right)\right)$ to $x^{\prime} \notin E$, for if $G$ is a $J^{\prime}$-open set containing $x^{\prime}$ then $X^{\prime}-G$ is J-countably compact, which implies that all but a finite number of terms of $S$ are in $G$. We conclude that $\mathfrak{J}^{\prime}$ is full.

The next result yields information on the embedding of mosaic spaces in compact mosaic spaces. 
5.4. Corollary. Let $(X, J)$ be a mosaic space and let $\left(X^{\prime}, J^{\prime}\right)$ be its one point countable compactification, then the following statements are equivalent:

(a) the space $\left(X^{\prime}, J^{\prime}\right)$ is compact;

(b) there exists a compact mosaic space $(Y, \mathrm{~s})$ such that $(X, J)$ is a subspace;

(c) for every $E \subset X$, if $E$ is J-countably compact then it is J-compact.

Proof. The implication (a) $\rightarrow$ (b) is obvious. By (1.8) the implication (b) $\rightarrow$ (c) follows easily. If (c) holds then the definition of $J^{\prime}$ becomes the following: $E$ is $J^{\prime}$-open if and only if $E$ is J-open or $X^{\prime}-E$ is J-closed and J-compact. But this is just the definition of the Alexandroff one point compactification of a space, and so (a) follows.

That there exists a mosaic space for which the conditions of (5.4) do not hold is given by the following standard example.

5.5. Example. Let $X$ be the set of all countable ordinal numbers, endowed with the order topology. This space is Hausdorff, first countable, and countably compact, but not compact [7, Chapter 5]. Since it is Hausdorff and first countable it is a mosaic space by (2.4).

By definition a subset $E$ of a topological space $(X, J)$ is conditionally countably compact if and only if every infinite subset of $E$ has a 3-accumulation point. It is, of course, desirable for every such set to have countably compact closure. The next theorem shows that the embedding of mosaic spaces in hereditary countably compact mosaic spaces is related to this property.

5.6. Тнеокем. Let $(X, J)$ be a mosaic space and let $\left(X^{\prime}, J^{\prime}\right)$ be its one point countable compactification. Then the following statements are equivalent:

(a) the space $\left(X^{\prime}, J^{\prime}\right)$ is an hereditary mosaic space;

(b) there exists an hereditary, countably compact mosaic space $(Y, \delta)$ such that $(X, J)$ is a subspace;

(c) the space $(X, \Im)$ is hereditary, and every conditionally countably compact subset has countably compact closure.

Proof. The implication (a) $\rightarrow$ (b) is obvious. Now let (b) hold. Then clearly $(X, J)$ is hereditary. Let $E \subset X$ be J-conditionally countably compact, and let $E^{\prime}$ and $E^{\prime \prime}$ be respectively the J-closure and the $S$-closure of $E$. In order to prove that $E^{\prime}$ is J-countably compact it is sufficient to show that $E^{\prime \prime} \subset E^{\prime}$, since $(Y, \delta)$ is countably compact. Let $x \in E^{\prime \prime}$, then by $(4.3)$ there exists a sequence $S$ such that $S_{J} \subset E$ and $S$ converges $(\Phi(s))$ to $x$ since $(Y, s)$ is hereditary. If $S_{J}$ is finite then obviously $x \in E \subset E^{\prime}$. If $S_{J}$ is infinite then it has a J-accumulation point. We can thereby construct a sequence $S^{\prime}$ such that $S^{\prime}<S$ and $S^{\prime}$ converges $(\Phi(\mathfrak{J}))$ to some $y \in X$. Then $S^{\prime}$ converges $(\Phi(\mathcal{S}))$ to $x$ since $S^{\prime}<S$, and $S^{\prime}$ converges $(\Phi(\S))$ to $y$ since $(X, \Im)$ is a subspace of $(Y, \S)$. By (1.6) we have $x=y$. Since $S_{I}^{\prime} \subset E$ it follows that $y \in E^{\prime}$, and we conclude that $E^{\prime \prime} \subset E^{\prime}$. Therefore (b) $\rightarrow$ (c). 
To show that (c) $\rightarrow$ (a) holds it is sufficient to prove that if $x^{\prime}$ is a $J^{\prime}$ accumulation point of $E \subset X^{\prime}$, then there is a sequence $S$ in $E$ which converges $\left(\Phi\left(J^{\prime}\right)\right)$ to $x^{\prime}$. If $x^{\prime} \in E$ this is obvious. Alternatively if $E \subset X$ then $E \cap G \neq 0$ for every $J^{\prime}$-open set $G$ which contains $x^{\prime}$. This implies that $E$ is contained in no J-countably compact set, and so the J-closure of $E$ is not countably compact. By (c) it follows that $E$ is not conditionally countably compact, and so there exists a countable set $F \subset E$ with no J-accumulation point. Let $S$ be a sequence such that $S_{j}=S_{k}$ if and only if $j=k$ and $S_{J}=F$. Then $S$ converges $\left(\Phi\left(J^{\prime}\right)\right)$ to $x^{\prime}$, and we conclude that $(\mathrm{c}) \rightarrow(\mathrm{a})$.

The following example gives an hereditary mosaic space which does not satisfy the conditions of (5.6).

5.7. Example. Let $X$ be the plane set $\{(x, y): 0 \leqq x \leqq 1 ; 0 \leqq y \leqq 1\}$, and define a topology $\Im$ by the following system of local bases: for each $(u, v) \in X_{0}$ $=\{(x, y): 0 \leqq x \leqq 1 ; y=0\}$ a local base consists of all sets of the form $\{(u, v)\} \cup\left(E-X_{0}\right)$, where $E$ is a neighborhood of $(u, v)$ in the usual metric topology; for each $(u, v) \in X-X_{0}$ a local base consists of the usual metric neighborhoods. Then $(X, J)$ is a first countable, Hausdorff space and is therefore an hereditary mosaic space. The set $X-X_{0}$ is conditionally countably compact but its closure, which is $X$, is not countably compact as $X_{0}$ is an infinite discrete set.

6. Separation properties. As previously noted, every mosaic space is a $T_{1}$-space. In general this cannot be strengthened, for we next construct a compact hereditary mosaic space which is not Hausdorff.

6.1. Example. Let $X$ be the union of a countable number of distinct $\operatorname{arcs} X_{n}$, which pairwise intersect in a single common end point $x_{0}$ only. Let this collection of arcs, each of which has the usual topology, determine the mosaic topology $J$ on $X$. Then each point $x \in X$ such that $x \neq x_{0}$ has the usual metric local base. A local base at $x_{0}$ consists of all sets of the form $\cup\left\{Y_{n}: n=1,2, \cdots\right\}$, where each $Y_{n}$ is a nondegenerate subinterval of $X_{n}$ which contains $x_{0}$. Then the mosaic space $(X, J)$ has the following properties: (i) it is hereditary and every conditionally countably compact subset has countably compact closure; (ii) every countably compact subset is compact; (iii) the space is not locally countably compact at $x_{0}$. Now let $\left(X^{\prime}, J^{\prime}\right)$ be the one point countable compactification of $(X, J)$. By (i) and (5.6) this space is an hereditary mosaic space; by (ii) and (5.4) it is compact; and by (iii) and a remark just preceding (5.3) it is not Hausdorff.

In addition, we remark that according to (5.1) this example provides a compact, nonHausdorff space for which each properly finer space is not countably compact, and thereby not compact. This answers affirmatively a question which has been raised by Vaidyanathaswamy [11, Preface].

7. Cartesian products. If two mosaic spaces $(X, J)$ and $(Y, \S)$ are first countable then clearly their Cartesian product $(X \times Y, J \times S)$ is a mosaic space. If the situation is made less stringent then the product need not be a 
mosaic space, for a result due to Dowker [5, page 563] shows that the product of the hereditary mosaic space $(X, J)$ of $(6.1)$ and the analogous hereditary mosaic space obtained by using a collection of arcs of the power of the continuum is not a mosaic space. Since obviously uniqueness of limits of convergent sequences carries over to the product of two mosaic spaces the difficulty lies in the fact that $J \times S$ may fail to be full. This leads to the mosaic space $(X \times Y, \Psi \Phi(J \times S))$, obtained by refining $J \times S$. We remark that this finer topology can be described as follows: let $\left\{\left(X_{a}, J_{a}\right): a \in A\right\}$ be any mosaic of compact metric spaces on $X$ which determines the topology $\mathfrak{J}$, and let $\left\{\left(Y_{b}, S_{b}\right): b \in B\right\}$ be any mosaic of compact metric spaces determining $\mathcal{S}$ on $Y$, then the mosaic of compact metric spaces $\left\{\left(X_{a} \times Y_{b}, J_{a} \times S_{b}\right): a \in A ; b \in B\right\}$ on $X \times Y$ determines the topology $\Psi \Phi(J \times S)$. This is the so-called weak product topology; see Cohen [2] for related material.

Given a fixed mosaic space, if the demand is made that its Cartesian product with any mosaic space be again a mosaic space, then necessary and sufficient conditions can be found. To this end there is exploited the fact that a mosaic space is the quasi-compact image of a suitable locally compact metric space; we proceed with a lemma which is a slight modification of a result due to Whitehead [12, Lemma 4].

7.1. Lemma. Let $f: P \rightarrow X$ be a quasi-compact function from the topological space $(P, \mathfrak{T})$ onto the topological space $(X, \mathfrak{J})$, and let $(Y, \S)$ be a topological space. Then the function $f \times i: P \times Y \rightarrow X \times Y$, defined by $(f \times i)(p, y)=(f(p), y)$ for all $(p, y) \in P \times Y$, is quasi-compact for the Cartesian product topologies on $P \times Y$ and $X \times Y$, provided either one of the following two conditions holds:

(a) the space $(Y, s)$ is locally compact and regular;

(b) the space $(Y, \delta)$ is a locally countably compact, regular mosaic space and $(P, \mathfrak{T})$ is first countable.

Proof. If (a) is assumed then the lemma is a special case of Whitehead's result. To prove the lemma for (b) we use the Whitehead method with a suitable modification. Let $E$ be an open subset of $P \times Y$ which is saturated with respect to $f \times i$, namely $(f \times i)^{-1}(f \times i)(E)=E$, and let $\left(x_{0}, y_{0}\right) \in(f \times i)(E)$. Then it is sufficient to show that there are open sets $U \subset P$ and $V \subset Y$ such that $U$ is saturated with respect to $f$ and $\left(x_{0}, y_{0}\right) \in f(U) \times V \subset(f \times i)(E)$. Let $p_{0} \in f^{-1}\left(x_{0}\right)$ and let $\left(\left\{p_{0}\right\} \times Y\right) \cap E=\left\{p_{0}\right\} \times Y_{0}$. Then $Y_{0}$ is an open subset of $Y$ for which $y_{0} \in Y_{0}$. By (b) there is an open subset $V \subset Y$ such that $y_{0} \in V$ and $\bar{V}$, the closure of $V$, is a countably compact subset of $Y_{0}$. Let $U$ be the set of all points $p \in P$ such that $\{p\} \times \bar{V} \subset E$. Then $p_{0} \in U$ and $U \times \bar{V} \subset E$, hence $\left(x_{0}, y_{0}\right) \in f(U) \times V=(f \times i)(U \times V) \subset(f \times i)(E)$. Since it can be shown that $U$ is saturated with respect to $f$ the lemma follows once it is shown that $U$ is open.

Now let $p \in U$, then $\{p\} \times \bar{V} \subset E$. Let $\left\{F_{i}: i=1,2, \cdots\right\}$ be a local base of open sets at $p$ in the first countable space $(P, \mathfrak{T})$. Assume for every $i$ that 
$F_{i} \times \bar{V} \nsubseteq E$, then there exists a sequence $S$ in $P \times Y$ such that $S_{J} \subset(P \times Y)-E$, $S^{P}$ converges $(\Phi(\mathscr{I}))$ to $p$ and $S_{J}^{Y} \subset \bar{V}$, where $S_{j}=\left(S_{j}^{P}, S_{j}^{Y}\right)$ for all $j \in J$. Since $\bar{V}$ is countably compact, by (1.7) we can extract a subsequence $T$ of $S$ such that $T^{Y}$ converges $(\Phi(S))$ to some $y \in \bar{V}$. Consequently $T$ converges in the product topology to $(p, y) \in\{p\} \times \bar{V} \subset E$. But $T$ is in the closed set $(P \times Y)$ $-E$ and so cannot have any limit in $E$. By this contradiction there exists an $F_{i}$ such that $F_{i} \times \bar{V} \subset E$. Hence $F_{i} \subset U$, and we conclude that $U$ is open.

7.2. Theorem. Let $(X, J)$ be a fixed mosaic space, then its Cartesian product with each mosaic space $(Y, \mathrm{~S})$ is a mosaic space if and only if $(X, \mathfrak{J})$ is locally countably compact and regular.

Proof. Let $(X, J)$ be a locally countably compact, regular mosaic space, and let $(Y, \S)$ be an arbitrary mosaic space. By (3.8) there exist locally compact metric spaces $(P, \mathfrak{T})$ and $(Q, \Re)$ such that $f: P \rightarrow X$ and $g: Q \rightarrow Y$, where $f$ and $g$ are quasi-compact and onto. Now define $f \times g: P \times Q \rightarrow X \times Y$ by $(f \times g)(p, q)=(f(p), g(q))$ for all $(p, q) \in P \times Q$. Since limits of sequences in $X \times Y$ which converge according to the product topology are clearly unique it follows from (3.7) and the fact that the product of two metric spaces is obviously a mosaic space that $X \times Y$ with the product topology is a mosaic space, once it is shown that $f \times g$ is quasi-compact.

Now write $f \times g=(i \times g)(f \times i)$ where $f \times i: P \times Q \rightarrow X \times Q$ and $i \times g$ : $X \times Q \rightarrow X \times Y$ are defined in the obvious way. By hypothesis (a) of (7.1) as applied to $(Q, \Re)$ the function $f \times i$ is quasi-compact; by hypothesis (b) of (7.1) as applied to $(Q, \Re)$ as the first countable space and $(X, \mathfrak{J})$ as the locally countably compact, regular mosaic space the function $i \times g$ is quasi-compact. Therefore $f \times g$ is quasi-compact, since it is the composition of two quasicompact functions.

Conversely, assume that $(X, J)$ is not locally countably compact. Let $\left(X^{\prime}, J^{\prime}\right)$ be its one point countable compactification, which is a mosaic space by (5.3). By a remark just preceding (5.3) this latter space is not Hausdorff; indeed, if $x^{\prime}$ is the point added to $X$ in order to obtain $X^{\prime}$, then there is an $x_{0} \in X$ such that for every pair of $J^{\prime}$-open sets $E_{1}$ and $E_{2}$ if $x_{0} \in E_{1}$ and $x^{\prime} \in E_{2}$ then $E_{1} \cap E_{2} \neq 0$. Let $X \times X^{\prime}$ be endowed with the product topology and define $D^{\prime} \subset X \times X^{\prime}$ by $D^{\prime}=\{(x, x): x \in X\}$. Then $\left(x_{0}, x^{\prime}\right)$ is an accumulation point of $D^{\prime}$ in the product topology, but $\left(x_{0}, x^{\prime}\right) \notin D^{\prime}$, and so $D^{\prime}$ is not closed. On the other hand, every sequence in $D^{\prime}$ which converges according to the product topology has its limit in $D^{\prime}$. Since the product topology is not full we conclude that $X \times X^{\prime}$ is not a mosaic space.

Alternatively, assume that $(X, J)$ is not regular, so that there exists a point $x_{0}$ and a closed set $F$ such that $x_{0} \notin F$ and for every pair of open sets $E_{1}$ and $E_{2}$ if $x_{0} \in E_{1}$ and $F \subset E_{2}$ then $E_{1} \cap E_{2} \neq 0$. In $X$ define an equivalence relation such that the equivalence classes are $F$ and all sets of the form $\{x\}$, where $x \notin F$. Let $\left(X^{\prime \prime}, J^{\prime \prime}\right)$ be the ensuing quotient space; since the projection 
function from $X$ onto the quotient space is closed and continuous it follows from (3.6) that $\left(X^{\prime \prime}, J^{\prime \prime}\right)$ is a mosaic space. Endow $X \times X^{\prime \prime}$ with the product topology, and define $D^{\prime \prime}=\{(x,\{x\}): x \in F\} \cup\{(x, F): x \in F\}$. Then $D^{\prime \prime}$ is not closed in the product topology, as $\left(x_{0}, F\right)$ is an accumulation point of $D^{\prime \prime}$ which is not contained in $D^{\prime \prime}$. In contradistinction, every convergent sequence in $D^{\prime \prime}$ has its limit in $D^{\prime \prime}$. We conclude that the product topology is not full, and so $X \times X^{\prime \prime}$ is not a mosaic space.

8. Function spaces. Let $(Y, \mathfrak{T})$ and $(Z, \mathfrak{T})$ be $\mathscr{L}^{*}$-spaces, and let $Z^{Y}$ be the class of sequentially continuous functions defined on $Y$ and taking values in $Z$. On $Z^{Y}$ a convergence class $\nVdash$ may be defined as follows: a sequence $R$ in $Z^{Y}$ converges $(\mathcal{K})$ to $f \in Z^{Y}$ if and only if for every sequence $S$ and point $y$ in $Y$ if $S$ converges (TT) to $y$ then the sequence $T$ defined by $T_{j}=R_{j}\left(S_{j}\right)$ for all $j \in J$ converges $(\mathfrak{T})$ to $f(y)$ in $Z$. This is the concept of continuous convergence for which Kuratowskl [8] has proved the following facts: (i) $\left(Z^{Y}, \mathfrak{K}\right)$ is an $\mathfrak{L}^{*}$-space; (ii) for any $\mathfrak{L}^{*}$-space $(X, \mathfrak{L})$ and any function $h: X \times Y \rightarrow Z, h$ is sequentially continuous for the product convergence class $\mathscr{L} \times \mathfrak{M}$ and $\mathscr{N}$ if and only if $h^{*}: X \rightarrow Z^{Y}$ is sequentially continuous for $\mathscr{L}$ and $\mathscr{K}$, where $h^{*}(x)(y)$ $=h(x, y)$ for all $x \in X, y \in Y$; (iii) the function $\omega: Z^{Y} \times Y \rightarrow Z$ defined by $\omega(f, y)=f(y)$ is sequentially continuous for $\mathscr{K} \times \mathfrak{N}$ and $\mathfrak{T}$. In general continuous convergence involving sequences is not equivalent to continuous convergence involving more sophisticated convergence objects, such as directed sets [1].

In contrast, let $(X, R),(Y, S)$ and $(Z, J)$ be mosaic spaces with their corresponding $\mathcal{L}_{u}^{*}$-spaces $(X, \Phi(R)),(Y, \Phi(S))$, and $(Z, \Phi(J))$. The fact that $S$ is a full topology guarantees that any function $f: Y \rightarrow Z$ is continuous for the topologies $S$ and $J$ if and only if it is sequentially continuous for the convergence classes $\Phi(S)$ and $\Phi(J)$; we may thereby identify the class of topologically continuous functions with the class of sequentially continuous functions. From $\Phi(S)$ and $\Phi(J)$ there is obtained the continuous convergence class $\mathscr{K}$ on the class of continuous functions $Z^{Y}$. Since the uniqueness of $\Phi(J)$-limits easily implies that $\mathcal{K}$-limits are unique, it follows that $\left(Z^{Y}, \mathcal{K}\right)$ is an $\mathfrak{L}_{u}^{*}$-space; this yields the corresponding mosaic space $\left(Z^{Y}, J_{M}\right)$, where $J_{M}=\Psi(\mathscr{K})$. As previously observed, the Cartesian product of two mosaic spaces need not be a mosaic space, but if this product is replaced with the weak product then Kuratowski's results for $\mathscr{L}^{*}$-spaces have exact analogs for mosaic spaces: (i') $\left(Z^{Y}, J_{M}\right)$ is a mosaic space; (ii') for any mosaic space $(X, R)$ and any function $h: X \times Y \rightarrow Z$, the function $h$ is continuous for the topologies $\Psi \Phi(R \times \&)$ $=\Psi(\Phi(R) \times \Phi(S))$ and $J$ if and only if $h^{*}: X \rightarrow Z^{Y}$ is continuous for the topologies $R$ and $\Im_{M}$; (iii') the function $\omega: Z^{Y} \times Y \rightarrow Z$ is continuous for $\Psi \Phi\left(J_{M} \times \delta\right)$ $=\Psi(\Re \times \Phi(\S))$ and $J$. In particular, if $(Y, \S)$ is locally countably compact and regular then by (7.2) weak products coincide with Cartesian products and $J_{M}$ is thereby entirely adequate as a function space topology in the sense that the continuity of $h$ is equivalent to the continuity of $h^{*}$ for mosaic spaces, 
without recourse to weak products. We now relate this material to the compact-open topology.

8.1. Lemma. Let $(Y, \mathcal{S})$ be a mosaic space and let $(Z, \Im)$ be a topological space. On the class $Z^{Y}$ of continuous functions from $Y$ to $Z$ let $\Im_{\text {co }}$ be the compactopen topology and let $\nVdash$ be the continuous convergence class derived from the convergence classes $\Phi(\mathcal{S})$ and $\Phi(\mathfrak{J})$. Then $\Phi\left(\mathfrak{J}_{C o}\right)=\mathcal{K}$.

Proof. Assume that $R$ does not converge $\left(\Phi\left(J_{C o}\right)\right)$ to $f \in Z^{Y}$. By the definition of the compact-open topology this is equivalent to the existence of a subsequence $R^{\prime}$ of $R$, a set $C$ which is $\delta$-compact, and a set $E$ which is J-open such that $f(C) \subset E$ and $R_{j}^{\prime}(C) \nsubseteq E$ for all $j \in J$. By (1.7) and the fact that $C$ is $S$-compact we can extract a subsequence $R^{\prime \prime}$ of $R^{\prime}$ and a sequence $S$ in $Y$ such that $R_{j}^{\prime \prime}\left(S_{j}\right) \notin E$ for all $j \in J$ and $S$ converges $(\Phi(s))$ to some $y \in C$. Since $f(y) \in E$ the sequence $R^{\prime \prime}$ does not converge (K) to $f$, and so $R$ does not converge $(\mathcal{K})$ to $f$. Conversely, if $R$ converges $\left(\Phi\left(J_{C O}\right)\right)$ to $f$ then it is known in general [7, page 241] that it converges $(\mathcal{K})$ to $f$.

The following theorem is a generalization of a result due to Fox [6, Theorem 2].

8.2. Theorem. Let $(X \times Y, R \times S)$ be a mosaic space. Then the function $h: X \times Y \rightarrow Z$, for any topological space $(Z, J)$, is continuous if and only if the corresponding function $h^{*}: X \rightarrow Z^{Y}$ is continuous, where $Z^{Y}$ is endowed with the compact-open topology.

Proof. In general the continuity of $h$ implies the continuity of $h^{*}$ [6, Lemma 1]. Conversely, assume that $h^{*}$ is continuous. Since $(X, \Re)$ is obviously homeomorphic to any closed subspace of $X \times Y$ of the form $X \times\{y\}$, $y \in Y$, it follows from (4.1) that it is a mosaic space, with the convergence class $\Phi(R)$. Now $h^{*}$ is sequentially continuous for the convergence classes $\Phi(R)$ and $\Phi\left(\Im_{C o}\right)$. By (8.1) we have $\Phi\left(\Im_{C o}\right)=\mathscr{K}$, and so by (ii) above $h$ is sequentially continuous for the convergence classes $\Phi(R) \times \Phi(S)=\Phi(R \times S)$ and $\Phi(J)$. Since $R \times S$ is a full topology by the mosaic hypothesis, $h$ is continuous for the topologies $R \times S$ and $J$.

8.3. Corollary. Let $Y$ be a locally countably compact, regular mosaic space, $X$ an arbitrary mosaic space, and $Z$ an arbitrary topological space. Then the continuity of $h^{*}: X \rightarrow Z^{Y}$, for $Z^{Y}$ cndowed with the compact-open topology, implies the continuity of $h: X \times Y \rightarrow Z$. Conversely, if $Y$ fails to be either locally countably compact or regular, then there exists a mosaic space $X$, a topological space $Z$ which is also a mosaic space, and a function $h$ such that $h^{*}: X \rightarrow Z^{Y}$ is continuous but $h: X \times Y \rightarrow Z$ is not continuous.

Proof. The first half of the corollary follows immediately from (7.2) and (8.2). Conversely, assume as one possibility that $Y$ is not locally countably compact. Let $X$ be the space $X^{\prime}$ of (7.2) and let $Z$ be $X^{\prime} \times Y$ endowed with 
the weak topology which is in this case properly finer than the Cartesian product topology. Choose $h: X^{\prime} \times Y \rightarrow Z$ to be the identity function, which is clearly not continuous. On the other hand, to show that $h^{*}: X^{\prime} \rightarrow Z^{Y}$ is continuous, where $h^{*}(x)(y)=(x, y)$, it is sufficient to prove that if $S$ converges in the topology on $X^{\prime}$ to $x$ then $h^{*} S$ is eventually in every sub-basic open set containing $h^{*}(x)$, as the topology on $X^{\prime}$ is full. By the definition of the compact-open topology in terms of sub-basic open sets and the fact that $h$ is the identity function this is equivalent to showing that if $S$ converges in the topology of $X^{\prime}$ to $x$ and $(x, C) \subset E$, where $C$ is a compact subset of $Y$ and $E$ is an open set of the weak product topology, then eventually $\left(S_{j}, C\right) \subset E$. Assume to the contrary that there is a subsequence $S^{\prime}$ of $S$ such that for each term we have $\left(S_{j}^{\prime}, C\right) \nsubseteq E$. Since $C$ is compact by (1.7) we may select a sequence $T$ in $\left(X^{\prime} \times Y\right)-E$ which converges in the Cartesian product topology, and so also in the weak product topology, to $(x, y)$, where $y \in C$. Since necessarily $(x, y) \in E$ the desired contradiction is reached, because $E$ being open in the weak product topology requires that $T$ be eventually in $E$.

Alternatively, if $Y$ is not regular let $X$ be the space $X^{\prime \prime}$ of (7.2) and repeat the argument just given.

The author wishes to thank the referee for suggestions which improved a number of the proofs.

\section{REFERENCES}

1. R. Arens and J. Dugundji, Topologies for function spaces, Pacific J. Math. vol. 1 (1951) pp. 5-31.

2. D. E. Cohen, Spaces with weak topology, Quart. J. Math. Oxford Ser. (2) vol. 5 (1954) pp. $77-80$.

3. - Products and carrier theory, Proc. London Math. Soc. (3) vol. 7 (1957) pp. 219-248.

4. W. F. Davison, An equivalence relation for compact Hausdorff varieties, Proc. Amer. Math. Soc. vol. 7 (1956) pp. 1109-1114.

5. C. H. Dowker, The topology of metric complexes, Amer. J. Math. vol. 74 (1952) pp. 555577.

6. R. H. Fox, On topologies for function spaces, Bull. Amer. Math. Soc. vol. 51 (1945) pp. $429-432$.

7. J. L. Kelley, General topology, van Nostrand, 1955.

8. C. Kuratowski, Sur la topologie des espaces fonctionnels, Anneles de la Société Polonaise de Mathématique vol. 20 (1947) pp. 314-322.

9. - Topologie I, Warsaw, 1948.

10. A. H. Stone, Metrizability of decomposition spaces, Proc. Amer. Math. Soc. vol. 7 (1956) pp. $690-700$.

11. R. Vaidyanathaswamy, Treatise on set topology, Indian Mathematical Society, 1947.

12. J. H. C. Whitehead, Note on a theorem due to Borsuk, Bull. Amer. Math. Soc. vol. 54 (1948) pp. 1125-1132.

UNIVERSITY OF VIRGINIA,

Charlottesville, Va.

University of Michigan,

Ann Arbor. Mich. 